\title{
A canonical semi-classical star product
}

\author{
Lucian M. IONESCU and Papa A. SISSOKHO \\ Illinois State University, Normal, IL 61790-4520, USA \\ E-mails:LMIones@ilstu.edu and psissok@ilstu.edu
}

\begin{abstract}
We study the Maurer-Cartan equation of the pre-Lie algebra of graphs controlling the deformation theory of associative algebras. We prove that there is a canonical solution (choice independent) within the class of graphs without circuits, i.e. at the level of the free operad, without imposing the Jacobi identity. The proof is a consequence of the unique factorization property of the pre-Lie algebra of graphs (tree operad), where composition is the insertion of graphs. The restriction to graphs without circuits, i.e. at "tree level", accounts for the interpretation as a semi-classical solution. The fact that this solution is canonical should not be surprising, in view of the Hausdorff series, which lies at the core of almost all quantization prescriptions.
\end{abstract}

2000 MSC: 53D55, 14Fxx

\section{Introduction}

The present article presents a combinatorial solution of the Maurer-Cartan equation in the Lie algebra of binary graphs $(g,[\cdot, \cdot])$ which controls the deformation theory of associative algebras:

$$
[Z, Z]=0, \quad Z=\sum_{\Gamma \in \mathcal{B}} \Gamma
$$

where $\mathcal{B}$ is the natural basis of graphs of $g$. The specific solution is "canonical" in the sense that it does not depend on choices made iteratively to extend the solution from one degree to the next, as in the cohomological context, nor it depends on a particular choice of contraction as in $[10]$.

The main result is that the underlying pre-Lie algebra multiplication, although not associative, has the unique factorization property. This in turn amounts to checking that the left and right Gerstenhaber composition multiplicity coefficients are equal.

The deformation quantization of Poisson manifolds was brilliantly solved by Kontsevich in a breakthrough article [14] which generated a "shower" of subsequent research papers.

Deformation quantization amounts, essentially, to the deformation of the usual classical commutative product of classical observables into a non-commutative star product, accommodating Heisenberg's canonical commutation relations corresponding to the given Poisson structure encoding the classical dynamics of the classical system. Kontsevich solution used the sophisticated machinery of perturbative quantum field theory, allowing to write the star product as a formal power series. The difficulty of finding such a "continuation" of the expansion starting with the commutative product as the zero term and the Poisson bracket as the first order term, resides in the requirement of achieving associativity. This amounts to the fact that the coefficients must satisfy a cocycle equation, which is combinatorial in nature. It is a fact that Kontsevich's coefficients $W_{\Gamma}$ [14] do not depend on the Poisson structure, and are Feynman integrals corresponding to any closed form, not just for the specific angle form, chosen for definiteness [15] (see also $[17,16])$.

In [18] it was claimed that the initial value deformation problem in the pre-Lie algebra of graphs has a canonical solution when restricted to graphs without circuits. It was first shown 
that the "coefficients problem" can be pulled back to the differential graded Lie algebra of graphs $([18], \S 5)$, which leads to a certain universal framework for deformation theory with the corresponding solutions of the Maurer-Cartan equation governing the space of deformations [1].

The claimed existence from [18] still relied on Kontsevich solution, i.e. on a star-product corresponding to a general Poisson structure which conjecturally yields a star-product when restricted to graphs without circuits. In [6] was realized that a different argument is needed to prove the existence of a solution when restricting the class of graphs (quasi-isomorphic complexes).

Further insight in the case of linear Poisson structures star products was given by S. Gutt $[7,8]$ and studied in the light of Kontsevich's approach by Polyak [21].

To get to the our main concern, the pre-Lie algebra of graphs, we will browse through Kontsevich's construction.

The combinatorial problem regarding the coefficients of a star-product is captured by the "graphical calculus" we will call Kontsevich rule, a sort of a "dual Feynman rule":

$$
B: k \mathcal{G} \rightarrow D, \quad B(\Gamma)=\mathcal{U}(\exp (\alpha))
$$

Here $\mathcal{G}$ is the class of graphs described below, $(D, \circ, m)$ is some pointed pre-Lie algebra [20] with a distinguished element $m$ such that $m \circ m=0$ and $\alpha$ is a Poisson structure (say on $R^{n}$ ):

$$
\alpha=\sum_{i, j} \alpha^{i j} \partial_{i} \otimes \partial_{j}
$$

It is an antisymmetric 2-tensor satisfying the Jacobi identity,

$$
\alpha^{i j}=-\alpha^{j i}, \quad \sum_{\text {circular }}\{\{f, g\}, h\}=0
$$

To a particular type of Poisson structure (e.g. constant/linear coefficients) corresponds a specific class of directed labeled graphs: those graphs $\Gamma$ which are not in the kernel of the Kontsevich rule $(k \mathcal{G} / \operatorname{Ker} K)$. Once the "Problem" is pull back to graphs, it amounts to solving the equation

$$
Z \circ Z=0, \quad Z=\sum_{n} Z_{n} h^{n}, Z_{n}=\sum_{\Gamma \in \mathcal{G}_{n, 2}} W_{\Gamma} \Gamma
$$

in a pre-Lie algebra with the composition o of [21], defined independently in [18].

In this article we prove that if the pre-Lie algebra has the unique factorization property, a canonical solution exists (Theorem 3.1), namely the "graph exponential"

$$
Z=\sum_{\Gamma} \Gamma /|A u t(\Gamma)|
$$

We develop general tools to investigate this property and we succeed to prove that this is the case for the class of Lie admissible graphs (Corollary 3.1). We also conjecture that the property is still enjoyed by graphs without circuits.

The proof relies on the result regarding the multiplicity coefficient for the the graph insertion at a boundary point as a pre-Lie operation (The Coefficient Theorem 3.1). A sort of Galois theory relating the symmetry of graphs with their extensions emerges. In this article we have dealt only with the simple case of unique incoming arrow case.

The article is organized as follows.

In Section 2 we recall the class of graphs [14] together with the pre-Lie composition from [18] (see also $[21,11])$. The core of the article is Section 3 which claims the "obvious solution" and introduces the main properties of graph insertion used in the subsequent proof.

In Section 4 we discuss some related questions. 


\section{The pre-Lie algebra of graphs}

\subsection{Lie admissible graphs}

Let $\mathcal{G}_{n, m}$ be the set of orientation classes of Lie admissible edge labeled graphs of [21], p. 3. An element $\Gamma \in \mathcal{G}_{n, m}$ is a directed graph with $n$ internal vertices, $m$ labeled boundary vertices $1,2, \ldots, m$ such that each internal vertex is trivalent with exactly two descendants. The corresponding arrows will be labeled left/right, defining the orientation class of the graph $\Gamma$ up to a transposition of the edge labeling in any two internal vertices [21]. The corresponding (graded set) is denoted by $\mathcal{G}=\cup \mathcal{G}_{m}$, where $\mathcal{G}_{m}=\cup_{n \in \mathbb{N}} \mathcal{G}_{n, m}$.

Note that this class of graphs, with only one incoming arrow at a node, corresponds to linear Poisson structures, i.e. in such a case, graphs with more than one incoming arrow would yield a zero contribution under the graphical calculus of derivations.

\subsection{Graphical representation and notation}

The order of the boundary vertices is "fixed" once and for all and will be represented graphically by placing the boundary vertices on a oriented line.

The left/right labels on the outgoing edges at each internal vertex are implicit in a graphical representation of a graph $\Gamma$, as embedded in the upper half-plane with edge ordering induced by counter clockwise orientation. To "brake the symmetry" of graphs like $c_{2}$ shown below, a labeling should be used instead, if needed (not present below).

The graphs from $\mathcal{G}_{n, 2}$ with $n=0,1,2$ internal vertices are the Bernoulli graphs $b_{0}, b_{1}, b_{2}^{L / R}$ or the products of Bernoulli graphs $\left(b_{1}^{2}=b_{1} \cdot b_{1}\right.$, to be defined shortly) as follows:

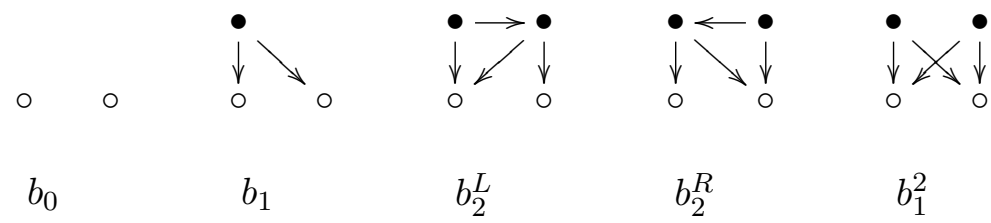

The "connected" graphs (see 2.4) from $\mathcal{G}_{n, 3}$ are represented below.
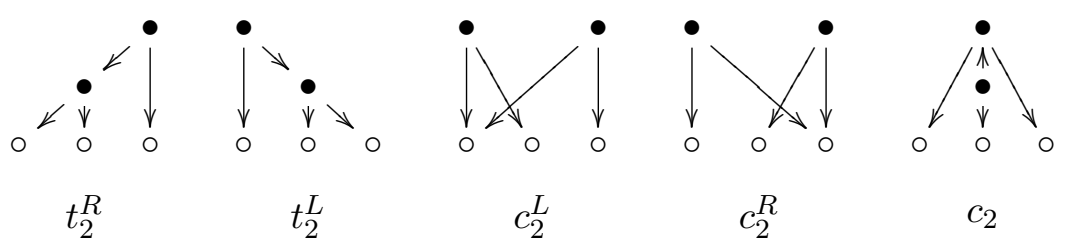

$c_{2}$

\subsection{Antisymmetry and Jacobi relation}

The Kontsevich rule has an obvious kernel since the Poisson tensor $\alpha$ is antisymmetric and satisfies the Jacobi identity.

Since we solve the Maurer-Cartan equation "on the nose", we do not assume the Jacobi relation being satisfied at the level of graphs.

We will only consider the anti-symmetry relation $\sim$ : a permutation of the edge order at an internal vertex is equivalent to a change of sign. This relation includes (is compatible) with the equivalence relation on edge labeling from the previous section $\S 2.2$. The resulting quotient is denoted by $H=k \mathcal{G} / \sim$. 


\subsection{Product of graphs}

The product of graphs $\mathcal{G}_{n, m} \times \mathcal{G}_{n^{\prime}, m} \rightarrow \mathcal{G}_{n+n^{\prime}, m}$ (L-graph multiplication [11], p. 23; [21], p. 3, [18], p. 5) is defined by identifying their corresponding boundary points (thought as embedded in the upper half-plane). A graph is prime if by "cutting its boundary" it yields a "graph" with only one component. For example, $b_{1}^{2}=\left(b_{1}\right)^{2}$, is not a prime graph.

Viewed as an "abstract graph" (incidence matrix), a graph is prime if the set of transition paths has more then one component. We will also call such a graph, connected.

Note that the product is compatible with the equivalence relation on edge-labeled graphs, and will be extended linearly on $H$.

The subspace generated by prime graphs is denoted by $g$. For this purpose the unit $b_{0}$ is considered prime.

\subsection{Composition of graphs}

The graph composition of [11] was introduced in [18] (p. 8) at the level of unlabeled graphs, as the pullback of the Gerstenhaber composition through Kontsevich rule (see also [21]). It acquires Leibniz rule in this process, since under Kontsevich representation the arrows carry differential operators, while boundary vertices are "colored" by functions. For example,

$$
b_{1}^{2} \circ b_{0}=b_{1}^{2} \circ_{1} b_{0}-b_{1}^{2} \circ_{2} b_{0}=\bullet b_{1}^{2}-b_{1}^{2} \bullet+2\left(c_{2}^{R}-c_{2}^{L}\right)
$$

where with $\bullet$ the only graph in $\mathcal{G}_{0,1}$ and $\bullet \Gamma$ denotes the "concatenation" of the corresponding graphs.

Note that graph composition is compatible with the grading by the number of boundary vertices (see [18], Appendix p. 22):

$$
\Gamma \in \mathcal{G}_{n, m}, \quad \operatorname{deg}_{b}(\Gamma)=m-1, \quad \operatorname{deg}_{b}\left(\Gamma_{1} \circ \Gamma_{2}\right)=\operatorname{deg}_{b}\left(\Gamma_{1}\right)+\operatorname{deg}_{b}\left(\Gamma_{2}\right)
$$

The above composition does not invary the class of Lie admissible graphs corresponding to linear Poisson structures. Since we are interested in the graphs not in the kernel of the Kontsevich representation, we will consider the truncation of the above composition due to the (orthogonal) projection $\operatorname{Pr}$ from all admissible graphs to our class of Lie admissible edge-labeled graphs $\mathcal{G}$. The resulting composition of graphs is now an internal operation, still graded by $\operatorname{deg}_{b}$.

Definition 2.1. The internal composition of graphs of $\mathcal{G}$ is defined as follows:

$$
\Gamma_{1} \circ \Gamma_{2}=\operatorname{Pr}\left[\sum_{i=1}^{m}(-1)^{(i-1)\left(m^{\prime}-1\right)} \Gamma_{1} \circ_{i} \Gamma_{2}\right], \quad \Gamma_{1} \in \mathcal{G}_{n, m}, \quad \Gamma \in \mathcal{G}_{n^{\prime}, m^{\prime}}
$$

where $\circ_{i}$ is the insertion of $\Gamma_{2}$ at the $i^{t h}$ boundary vertex of $\Gamma_{1}$ using "Leibniz rule" i.e. summing over all possible graphs where the " $i$ th legs" of $\Gamma_{1}$ lend on vertices of $\Gamma_{2}$, internal and external. The edge-labeling of the resulting graph is inherited from the edge-labeling of the two graphs $\Gamma_{i}$.

Graph composition is compatible with the anti-symmetry relation $\sim$, inducing a graph composition on $H$ ([21], p.5).

If $I_{i}$ denotes the set of incoming edges at the $i^{t h}$ boundary point of $\Gamma_{1}$ and $\left[n_{2}\right],\left[m_{2}\right]$ denote the sets of internal and respectively external vertices of $\Gamma_{2}$, then the "Leibniz rule" at the $i^{t h}$ vertex yields

$$
\Gamma_{1} \circ_{i} \Gamma_{2}=\sum_{f: I_{i} \rightarrow\left[n_{2}\right] \cup\left[m_{2}\right]} \Gamma_{1} \circ_{i}^{f} \Gamma_{2}
$$


where $\circ_{i}^{f}$ denotes the operation of replacing the vertex $i$ with a disjoint copy of the set $\left[n^{\prime}\right]$, with the edges $e=(v \rightarrow i) \in I_{i}$ now pointing to $f(e)$. The "two components" of $f, f_{i}, f_{b}$ denote their co-restriction to internal and boundary vertices, respectively.

Now in order for the resulting graph to have internal vertices with only one incoming arrow, the component $f_{i}$ must be injective, yielding the following formula for graph composition.

Lemma 2.1. If $\Gamma_{1} \in \mathcal{G}_{n_{1}, m_{1}}$ and $\Gamma_{2} \in \mathcal{G}_{n_{2}, m_{2}}$, then

$$
\Gamma_{1} \circ_{i} \Gamma_{2}=\sum_{f_{i} \cup f_{b}: I_{i} \hookrightarrow\left[n_{2}\right] \cup\left[m_{2}\right]} \Gamma_{1} \circ_{i}^{f} \Gamma_{2}
$$

where $f_{i}$ is $1: 1$.

We are now ready to prove that the "sum of all graphs" is a solution.

\section{The canonical solution}

We will study a particular solution of $[Z, Z]=0$ in the graded pre-Lie algebra of graphs, with possibly multiple incoming arrows at internal nodes. The tools developed apply to this level of generality, yet we have been able to prove the key result of unique factorization only for Lie admissible graphs (only one incoming arrow at a node).

Let $Z_{k}=\sum_{\Gamma \in \mathcal{G}_{k, 2}} \Gamma /|A u t(\Gamma)|$ and $Z=\sum Z_{k} h^{k}$. In order to prove $Z \circ Z=0$ we need to investigate the coefficients of

$$
\sum_{i+j=n ; i, j \geq 0} Z_{i} \circ_{1} Z_{j}-Z_{i} \circ_{2} Z_{j}=\sum_{\Gamma \in \mathcal{G}_{n, 3}} B_{\Gamma} \cdot \Gamma
$$

where the coefficient $B_{\Gamma}$ is the difference between the coefficients (possibly zero) of the graph $\Gamma$ resulting from left and from right graph insertions $\left(\circ_{1}\right.$ and $\left.\circ_{2}\right): B_{\Gamma}=B_{\Gamma}^{L}-B_{\Gamma}^{R}$. To simplify notation, for any $\Gamma \in \mathcal{G}_{n, m}, \underline{\Gamma}$ denotes the corresponding normalized basis element, i.e. $\Gamma /|A u t(\Gamma)|$. The normalized bases of $k G$ is $\{\underline{\Gamma}\}_{\Gamma \in \mathcal{G}_{n, m}}$.

The key fact (Proposition 3.1) is that $\circ_{1}$ is "injective" (similarly $\circ_{2}$ ), i.e. from the composition $\Gamma_{1} \circ_{1} \Gamma_{2}$ one can recover the operands $\Gamma_{1}$ and $\Gamma_{2}$ ("left groupoid structure" $\Gamma: \Gamma_{1} \rightarrow \Gamma_{2}$ ). In general the pair $\left(\Gamma_{1}, \Gamma_{2}\right)$ responsible for a summand $\Gamma$ as a result of a left insertion $\circ_{1}$ is different from the unique pair yielding a sum involving $\Gamma$ in a right insertion $\mathrm{O}_{2}$ (is the "left groupoid" isomorphic to the "right groupoid"?).

Now comparing the two sums

$$
\Sigma_{k}=\sum_{i+j=n ; i, j \geq 0} \sum_{\Gamma_{1} \in \mathcal{G}_{i, 2} ; \Gamma_{2} \in \mathcal{G}_{j, 2}} \Gamma_{1} \circ_{k} \Gamma_{2}, \quad k=1,2
$$

corresponding to left, and respectively right insertions, we obtain that the respective coefficients are equal (Corollary 3.3), a fact expected due to the left/right symmetry, and proved as The Coefficient Theorem 3.1.

Theorem 3.1. If $Z=\sum_{\Gamma \in \mathcal{G}_{n, 2}} \underline{\Gamma}$, then $[Z, Z]=0$.

To prove the above claims, we start with some preparatory lemmas.

For $\Gamma \in \mathcal{G}$, let $V^{\text {in }}(\Gamma)$ denote its set of internal vertices, $V^{\text {bd }}(\Gamma)$ its set of boundary vertices, and let $V(\Gamma)=V^{\text {in }} \cup V^{\text {bd }}$. For $u \in V^{\text {in }}(\Gamma)$, let $u_{L}$ and $u_{R}$ be the left and right descendants of $u$, respectively. Moreover, denote by $(u, \ldots, v)$ a directed path starting at $u$ and ending at the vertex $v$. 
Lemma 3.1. Let $\Gamma \in \mathcal{G}_{n, 2}$ with boundary vertices $L$ and $R$. Then for each internal vertex $u$ of $\Gamma$, there is a directed path from $u$ to $L$ and a directed path from $u$ to $R$

Proof. Define a partial order on internal vertices corresponding to the "flow" direction corresponding to the oriented edges (no loops!). Since any internal vertex has two descendants, clearly there is a path starting at $u$ ending at a boundary point, say $L$. Now not all paths may end at $L$, since one may trace back last arrow and descend on the other arrow, until the end of the path is not $L$.

In particular, binary graphs without loops are connected.

Remark 3.1. Note that the lemma may fail for graphs with loops, and for $m=0,1, \mathcal{G}_{n, m}$ contains no binary admissible graph without loops.

Lemma 3.2. Let $\Gamma \in \mathcal{G}_{n, 3}$ and $\Theta$ be a normal subgraph of $\Gamma$, i.e. $\Gamma / \Theta$ is still admissible, with at least one boundary point.

(i) If $u \in V^{\text {in }}(\Theta)$ then $u_{L}, u_{R} \in V(\Theta)(\Theta$ is a "total subgraph" of $\Gamma)$.

(ii) If $\left(v_{1}, \ldots, v_{t}\right)$ is a path from an interior point $v_{1}$ of $\Theta$ to a boundary point $v_{t}$ of $\Gamma$ then $v_{i} \in V(\Theta)$ for all $1 \leq i \leq t(\Theta$ is "geodesically complete").

Proof. $(i)$ follows from the fact that if $u \in V(\Theta)$ and say $u_{L} \notin V(\Theta)$, then the edge $\left(u, u_{L}\right)$ is not present in $\Theta$. This would contradict that $\Theta$ is a binary graph, since it has an internal vertex $u$ with at most one outgoing edge. (ii) follows from a recursively application of $(i)$, using Lemma 3.1.

Our next goal is to prove that for each graph $\Gamma \in \mathcal{G}_{n, 3}$ there is a unique factorization in terms of graphs with less boundary points: $\Gamma=\Gamma_{1} \circ_{1} \Gamma_{2}\left(\Gamma=\Gamma_{1}^{\prime} \circ_{2} \Gamma_{2}^{\prime}\right)$. Each such decomposition will correspond to a "maximal factor" of $\Gamma$, so here too "maximal implies prime"!

Lemma 3.3. For $\Gamma \in \mathcal{G}_{n, 3}$ there are unique normal subgraphs of $\Gamma$, denoted $\alpha_{L}(\Gamma), \alpha_{R}(\Gamma) \in \mathcal{G}_{n, 2}$, sitting on the leftmost, and respectively rightmost, two boundary vertices of $\Gamma$.

Proof. Recall that being normal ensures that the quotient $\Gamma / \alpha_{L}(\Gamma)$ is still a binary (exactly two descendants) admissible graph.

Suppose that $\Gamma_{1}$ and $\Gamma_{2}$ are two different normal subgraphs of $\Gamma$ sitting on boundary points 1 and 2 of $\Gamma$ (the other case follows by symmetry): $b_{0}^{L}=\{1,2\} \subset \Gamma_{i} \subset \Gamma$. Then there exist an internal vertex $u$ of $\Gamma_{1}$ but not in $\Gamma_{2}$, since they cannot both equal $b_{0}^{L}$. Note that by Lemma 3.2 , any path starting at $u$ must end at a boundary vertex: 1 or 2 .

Since $\Gamma_{2}$ is normal, $\Gamma_{2}^{\prime}=\Gamma / \Gamma_{2} \in \mathcal{G}_{n, 2}$ is a binary admissible graph. By definition, we have $u \in V^{\text {in }}\left(\Gamma_{2}^{\prime}\right)$. However, there is no directed path from $u$ to the right boundary vertex $\Gamma_{2}^{\prime}$, contradicting Lemma 3.1.

Definition 3.1. For $\Gamma \in \mathcal{G}_{n, 3}$,

(i) $E_{\Gamma}^{L}=\underline{\left(\Gamma / \alpha_{L}(\Gamma)\right)} \circ_{1} \underline{\alpha_{L}(\Gamma)}$ and $E_{\Gamma}^{R}=\underline{\left(\Gamma / \alpha_{R}(\Gamma)\right)} \circ_{2} \underline{\alpha_{R}(\Gamma)}$,

(ii) $C_{\Gamma}^{L}=<E_{\Gamma}^{L}, \Gamma>$ and $C_{\Gamma}^{R}=<E_{\Gamma}^{R}, \Gamma>$ are the coefficients of $\Gamma$ in $E_{\Gamma}^{L}$, and $E_{\Gamma}^{R}$ respectively,

(iii) $C_{\Gamma}=C_{\Gamma}^{L}-C_{\Gamma}^{R}$.

We now prove the key fact, that the left and right insertions are "injective".

Proposition 3.1. Let $\Gamma^{\prime}, \Gamma^{\prime \prime} \in \mathcal{G}_{\bullet}, 2$ and $\Gamma \in \mathcal{G}_{n, 3}$.

(i) If $<\Gamma^{\prime \prime} \circ_{1} \Gamma^{\prime}, \Gamma>\neq 0$, then $\Gamma^{\prime}=\alpha_{L}(\Gamma)$ and $\Gamma^{\prime \prime}=\Gamma / \alpha_{L}(\Gamma)$.

(ii) If $<\Gamma^{\prime \prime} \circ_{2} \Gamma^{\prime}, \Gamma>\neq 0$, then $\Gamma^{\prime}=\alpha_{R}(\Gamma)$ and $\Gamma^{\prime \prime}=\Gamma / \alpha_{R}(\Gamma)$. 
Proof. Let $\Gamma^{\prime}, \Gamma^{\prime \prime} \in \mathcal{G}_{n, 2}$ be such that $\Gamma \in \mathcal{G}_{n, 3}$ is a summand of $\Gamma^{\prime \prime} \circ_{1} \Gamma^{\prime}$. Then, there exists a way to land legs from the left boundary vertex of $\Gamma^{\prime \prime}$ onto the vertices of $\Gamma^{\prime}$ so that the resulting graph is $\Gamma$.

Since $\Gamma^{\prime}$ is a normal subgraph of $\Gamma$ sitting on the its 1st and 2nd boundary vertex, it follows that $\Gamma^{\prime} \subseteq \alpha_{L}(\Gamma)$. Moreover $\Gamma^{\prime}$ is the maximal subgraph of $\Gamma$ sitting on its 1st and 2nd boundary vertex. For otherwise, $\alpha_{L}(\Gamma)$ contains an internal vertex $u$ of $\Gamma^{\prime \prime}$. Now, by Lemma 3.1, there exist a path $P_{u}^{R}$ from $u$ to the second boundary vertex of $\Gamma^{\prime \prime}$. By Lemma 3.2, all the vertices (internal and external) in $P_{u}^{R}$ are in $\alpha_{L}(\Gamma)$. In particular, the second boundary vertex of $\Gamma^{\prime \prime}$ (which is the third boundary vertex of $\Gamma$ ) would also have to be in $\alpha_{L}(\Gamma)$. This would contradict the fact that $\alpha_{L}(\Gamma)$ has to sit on the 1st and 2nd boundary vertex of $\Gamma$. Thus $\Gamma^{\prime}=\alpha_{L}(\Gamma)$.

Since $\Gamma$ is a summand of $\Gamma^{\prime \prime} \circ_{1} \Gamma^{\prime}$ and $\Gamma^{\prime}=\alpha_{L}(\Gamma)$, we have $\Gamma^{\prime \prime}=\Gamma / \alpha_{L}(\Gamma)$; because $o_{1}$ splits the edges landing on the 1st boundary vertex of $\Gamma^{\prime \prime}$ and land them on vertices of $\Gamma^{\prime}=\alpha_{L}(\Gamma)$ while collapsing $\alpha_{L}(\Gamma)$ in $\Gamma$ does the converse, recovering $\Gamma^{\prime \prime}$.

Similarly, if $\Gamma^{\prime}, \Gamma^{\prime \prime} \in \mathcal{G}_{n, 2}$ are such that $\Gamma$ is a summand of $\Gamma^{\prime \prime} \circ_{2} \Gamma^{\prime}$, then $\Gamma^{\prime \prime}=\alpha_{R}(\Gamma)$ and $\Gamma^{\prime}=\Gamma / \alpha_{R}(\Gamma)$.

Regarding graph insertions as partially defined binary operations, the above result may be rephrased as follows.

Corollary 3.1. Boundary graph insertions have the unique factorization property.

As an immediate consequence we obtain that the $\Gamma$-coefficients of $[Z, Z]$ result from a unique left/right composition, namely the composition of the unique normal maximal left/right supported subgraphs.

Corollary 3.2. $B_{\Gamma}=C_{\Gamma}$.

Proof. As a consequence of Proposition 3.1, $B_{\Gamma}=B_{\Gamma}^{L}-B_{\Gamma}^{R}$ represents the contributions from a left composition of a unique pair of graphs $\left(\Gamma_{1}, \Gamma_{2}\right)$ and of a right composition of a unique pair $\left(\Gamma_{1}^{\prime}, \Gamma_{2}^{\prime}\right)$. The corresponding multiplicities are $C_{\Gamma}^{L}$ and $C_{\Gamma}^{R}$. Therefore $B_{\Gamma}=C_{\Gamma}$.

All that is left in order to prove the main theorem, is to prove that left insertions produce the same coefficients as right insertions, i.e. $C_{\Gamma}^{L}=C_{\Gamma}^{R}$. Fix a summand $\Gamma$ of a fixed pair of graphs $\Gamma_{1}, \Gamma_{2}$, i.e. $\Gamma$ has a non-trivial coefficient $C_{\Gamma}^{L}$ in the sum expressing the left boundary composition $\Gamma_{1} \circ_{1} \Gamma_{2}$. Then there is a left extension $\Gamma_{2} \stackrel{\pi}{\rightarrow} \Gamma \rightarrow \Gamma_{1}$, characterized by the insertion data $\pi$ (see section 3.2 for additional details), with $\Gamma_{2}$ collapsing to the left boundary vertex of $\Gamma_{1}$.

The Coefficient Theorem 3.1. After normalization, the non-trivial coefficient of $\Gamma$ as a summand of the left insertion operation of $\Gamma_{2}$ in $\Gamma_{1}$ is 1. Therefore, if non-trivial, the left/right normalized multiplicities are

$$
\underline{L}_{\Gamma_{1} \Gamma_{2}}^{\Gamma}=<\underline{\Gamma_{1}} \circ_{1} \underline{\Gamma_{2}}, \underline{\Gamma}>=1=<\underline{\Gamma_{1}} \circ_{1} \underline{\Gamma_{2}}, \underline{\Gamma}>=\underline{R_{\Gamma_{1} \Gamma_{2}}^{\Gamma}}
$$

where $\pi_{L}, \pi_{R}$ are the left/right insertion data determined by the graph $\Gamma$.

We will first exploit the result, deferring the proof to section 3.2.

Corollary 3.3. (i) For all $\Gamma \in \mathcal{G}_{n, 3}$, its left multiplicity equals its right multiplicity: $C_{\Gamma}^{L}=C_{\Gamma}^{R}$. (ii) $\forall \Gamma \in \mathcal{G}_{\bullet}, 3$, we have $\left\langle\Sigma_{1}, \Gamma>=<\Sigma_{2}, \Gamma>\right.$.

Proof. Any $\Gamma \in \mathcal{G}_{n, 3}$ appears as part of $\left(\Gamma / \alpha_{L}(\Gamma)\right) \circ_{1} \alpha(\Gamma)$. The coefficient of $\Gamma$ in both $\Sigma_{1}$ and $\Sigma_{2}$ (Equation 3.1) is $|A u t(\Gamma)|$, i.e (i) holds, and the two sums are equal. 
It follows from Lemma 3.2 that $B_{\Gamma}=C_{\Gamma}$, which by the Corollary 3.3 vanish for all graphs $\Gamma$. This implies that

$$
\sum_{i+j=n ; i, j \geq 0} Z_{i} \circ Z_{j}=0
$$

which yields $Z \circ Z=0$, concluding the proof of the Main Theorem 3.1.

\subsection{Examples}

Consider the graphs $\Gamma_{1}, \Gamma_{2}, \Gamma_{3} \in \mathcal{G}_{1,3}$, defined as follows:

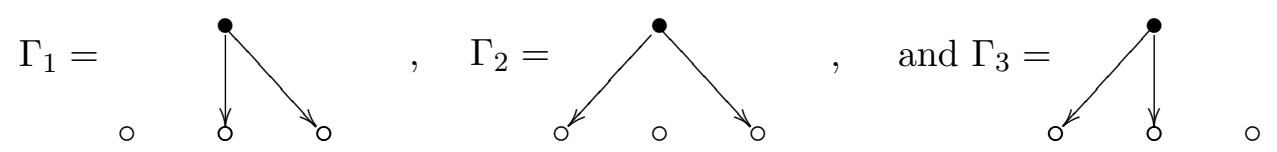

(1) The constant Case. Any admissible graphs $\Gamma \in \mathcal{G}_{n, 3}$ can be expressed as $\Gamma=\Gamma_{1}^{r} \Gamma_{2}^{s} \Gamma_{3}^{t}$, where $\Gamma_{i}, i=1,2,3$, is as defined earlier. Thus

$$
E_{\Gamma}=\underline{b_{1}^{r+s}} \circ_{1} \underline{b_{1}^{t}}-\underline{b_{1}^{s+t}} \circ_{2} \underline{b_{1}^{r}}
$$

Since the coefficient of $\Gamma$ in $\underline{b_{1}^{r+s}} \circ_{1} \underline{b_{1}^{t}}$ is $\left(\begin{array}{c}r+s \\ s\end{array}\right)$ and the coefficient of $\Gamma$ in $\underline{b_{1}^{s+t}} \circ_{2} \underline{b_{1}^{r}}$ is $\left(\begin{array}{c}s+t \\ s\end{array}\right)$ then

$$
C_{\Gamma}=\frac{\left(\begin{array}{c}
r+s \\
s
\end{array}\right)}{t !(r+s) !}-\frac{\left(\begin{array}{c}
s+t \\
s
\end{array}\right)}{r !(s+t) !}=0
$$

In the normalized bases $\underline{C}_{\Gamma}^{L}=\underline{C}_{\Gamma}^{R}=1$ (similarly for the other normalized coefficients below).

(2) The linear case with $n=2$. There are 9 admissible graphs in $\mathcal{G}_{2,3}: \Gamma_{1}^{2}, \Gamma_{2}^{2}, \Gamma_{3}^{2}, \Gamma_{1} \Gamma_{3}$, $t_{2}^{L}, t_{2}^{R} c_{2}^{L}, c_{2}^{R}$, and $c_{2}$.

For example, let $\Gamma=\Gamma_{1}^{2}$. We have

$$
\alpha_{L}(\Gamma)=b_{0}, \quad \alpha_{R}(\Gamma)=b_{1}^{2}, \quad \Gamma / \alpha_{L}(\Gamma)=b_{1}^{2}, \quad \text { and } \quad \Gamma / \alpha_{R}(\Gamma)=b_{0}
$$

Thus, the coefficient of $\Gamma$ is $b_{1}^{2} \circ_{1} b_{0}-\underline{b_{0}} \circ_{2} b_{1}^{2}$, which is $C_{\Gamma}=\frac{1}{2}-\frac{1}{2}=0$.

Similar computations take care of the other cases, yielding

$$
\sum_{i+j=2 ; i, j \geq 0}\left[Z_{i}, Z_{j}\right]=\sum_{\Gamma \in \mathcal{G}_{2,3}} C_{\Gamma} \cdot \Gamma=0
$$

\subsection{Proof of Theorem 3.1}

We prove that the multiplicity of a graph as a summand in a graph composition is only due to their groups of symmetries.

Fix graphs $\Gamma_{1}, \Gamma_{2}$ and a summand $\Gamma$ of their left boundary insertion as follows:

$$
L_{\Gamma_{1} \Gamma_{2}}^{\Gamma}=<\Gamma_{1} \circ_{1} \Gamma_{2}, \Gamma>\neq 0
$$

Since similar considerations apply to right insertions and to the corresponding coefficient $R_{\Gamma_{1} \Gamma_{2}}^{\Gamma}$, we will use the generic notation $C_{\Gamma_{1} \Gamma_{2}}^{\Gamma}$.

Then there is a left graph extension $\Gamma_{2} \stackrel{\pi}{\rightarrow} \Gamma \rightarrow \Gamma_{1}$, determined by the left insertion data $\pi: S \subset V_{1} \rightarrow T \subset V_{2}$ defining the way the left leg arrows of $\Gamma_{1}$ land on the vertices of $\Gamma_{2}$, internal or boundary. Each insertion data $\pi$ yields an admissible graph $\Gamma_{\pi}$. Its isomorphism class will be called the type of the insertion. 
Recall that for a linear Poisson structure, the non-boundary portion of the insertion $\pi$ is injective.

Let $\mathcal{D}$ be the set of all insertion data $\pi$. For any $\pi \in \mathcal{D}$, let $\mathcal{D}_{\Gamma} \subseteq \mathcal{D}$ be those insertion data of the same type as $\Gamma$. Then

$$
C_{\Gamma_{1} \Gamma_{2}}^{\Gamma}=<\Gamma_{1} \circ_{1} \Gamma_{2}, \Gamma>=\left|\mathcal{D}_{\Gamma}\right|
$$

For any insertion data $\pi$, let $A u t\left(\Gamma_{\pi}, \pi\right)$ be the set of automorphism $A u t\left(\Gamma_{\pi}\right)$ that fix $\pi$.

We claim that the multiplicity of a summand in a left (right) boundary composition is given by the following formula.

Lemma 3.4. We have

$$
\left|\mathcal{D}_{\Gamma}\right|=\frac{\left|A u t\left(\Gamma_{1}\right)\right| \cdot\left|A u t\left(\Gamma_{2}\right)\right|}{\left|A u t\left(\Gamma_{\pi}, \pi\right)\right|}
$$

We delay the proof of Lemma 3.4 to make some general observations.

Consider the action $\tau$ of $H=\operatorname{Aut}\left(\Gamma_{1}\right) \times \operatorname{Aut}\left(\Gamma_{2}\right)$ on $\mathcal{D}_{\Gamma}$ defined as follows. For all $\rho=$ $\left(\rho_{1}, \rho_{2}\right) \in H$ and for all $\pi \in \mathcal{D}$ with $\pi: S \subset V_{1} \rightarrow T_{\pi} \subset V_{2}$, we have

$$
\begin{aligned}
\tau(\rho, \pi)=\pi_{\rho}: \rho_{1}(S)=S & \rightarrow \rho_{2}\left(T_{\pi}\right) \\
x & \mapsto \rho_{2} \pi \rho_{1}^{-1}(x)
\end{aligned}
$$

Claim 3.1. For $\pi \in \mathcal{D}$, we have $\mathcal{O}(\pi)=\left\{\pi_{\rho}: \rho \in H\right\}=\mathcal{D}_{\Gamma_{\pi}}$, i. e the action $\tau$ is transitive on $\mathcal{D}_{\Gamma_{\pi}}$.

Proof. By definition, $\mathcal{O}(\pi) \subseteq \mathcal{D}_{\Gamma_{\pi}}$. Conversely, if $\Gamma_{\pi^{\prime}} \in \mathcal{D}_{\Gamma_{\pi}}$ then there exits $\pi^{\prime} \in \mathcal{D}$ such that $\Gamma_{\pi^{\prime}} \cong \Gamma_{\pi}$. Hence, there exist $\phi \in \operatorname{Aut}\left(\Gamma_{\pi}\right)$ such that $\phi\left(\mathcal{D}_{\Gamma_{\pi}}\right)=\Gamma_{\pi^{\prime}}$. Now let $\rho_{\phi}=\left(\phi_{\mid \Gamma_{1}}, \phi_{\mid \Gamma_{2}}\right) \in$ $H$; then $\rho_{\phi}(\pi)=\pi^{\prime} \in \mathcal{O}(\pi)$. Thus $\mathcal{D}_{\Gamma_{\pi}} \subseteq \mathcal{O}(\pi)$, and the claim follows.

Claim 3.2. For $\pi \in \mathcal{D}$, we have

$$
|\operatorname{Stab}(\pi)|=\left|\left\{\rho \in H: \pi_{\rho}=\pi\right\}\right|=\left|\operatorname{Aut}\left(\Gamma_{\pi}, \pi\right)\right|
$$

Proof. We show that there exist a bijection $f: \operatorname{Aut}\left(\Gamma_{\pi}, \pi\right) \rightarrow \operatorname{Stab}(\pi)$. For $\phi \in \operatorname{Aut}\left(\Gamma_{\pi}\right)$, define $f(\phi)=\left(\rho_{1}, \rho_{2}\right)$ by first restricting $\phi$ to the unique normal subgraph $\Gamma_{1}$, which therefore is invaried by $\rho_{1}(\phi)=\phi_{\mid \Gamma_{1}}$ Then, $\phi$ induces an automorphism of the quotient,

$$
\rho_{2}(\phi)=\phi_{\mid\left(\Gamma / \Gamma_{1}\right)}=\phi_{\mid \Gamma_{2}}
$$

Thus, $f(\phi)=\left(\rho_{1}, \rho_{2}\right) \in \operatorname{Stab}(\pi)$ since, by definition of $\rho_{1}$ and $\rho_{2} ., \rho_{2} \pi \rho_{1}^{-1}=\pi$. It is easy to see that $f$ is injective, since $V=V_{1} \cup V_{2}$.

To prove that $f$ is surjective, let $\rho=\left(\rho_{1}, \rho_{2}\right) \in \operatorname{Stab}(\pi)$. Then there exist unique automorphisms $\phi_{1}, \phi_{2} \in \operatorname{Aut}\left(\Gamma_{\pi}\right)$ obtained by extending $\rho_{1}$ and $\rho_{2}$ in such a way that $\phi_{1 \mid \Gamma_{2}}=i d_{\Gamma_{2}}$ and $\phi_{2 \mid \Gamma_{1}}=i d_{\Gamma_{1}}$. Thus $\phi=\phi_{1} \phi_{2} \in \operatorname{Aut}\left(\Gamma_{\pi}, \pi\right)$ is such that $\phi_{\mid \Gamma_{1}}=\rho_{1}$ and $\phi_{\mid \Gamma_{2}}=\rho_{2}$, i.e. $f(\phi)=\left(\rho_{1}, \rho_{2}\right)$. Since $f$ is a bijection, we have $|\operatorname{Stab}(\pi)|=\left|\operatorname{Aut}\left(\Gamma_{\pi}, \pi\right)\right|$.

Proof of Lemma 3.4. Using an orbit-stabilizer argument, (3.2), and Claims 3.1 and 3.2, we obtain

$$
C_{\Gamma_{1} \Gamma_{2}}^{\Gamma}=\mathcal{D}_{\Gamma_{\pi}}=|\mathcal{O}(\pi)|=\frac{|H|}{|\operatorname{Stab}(\pi)|}=\frac{\left|A u t\left(\Gamma_{1}\right)\right| \cdot\left|A u t\left(\Gamma_{2}\right)\right|}{\left|A u t\left(\Gamma_{\pi}, \pi\right)\right|},
$$

proving Lemma 3.4. 
Proof of The Coefficient Theorem 3.1. For $\Gamma \in \mathcal{G}_{n, 3}$, let

$$
\Gamma_{1}^{L}=\alpha_{L}(\Gamma), \quad \Gamma_{2}^{L}=\Gamma / \Gamma_{1}^{L}, \quad \Gamma_{1}^{R}=\alpha_{R}(\Gamma), \quad \text { and } \quad \Gamma_{2}^{R}=\Gamma / \Gamma_{1}^{R}
$$

Recall that

$$
C_{\Gamma}^{L}=<\underline{\Gamma}_{1}^{L} \circ_{1} \underline{\Gamma}_{2}^{L}, \Gamma>, \quad C_{\Gamma}^{R}=<\underline{\Gamma}_{1}^{R} \circ_{2} \underline{\Gamma}_{2}^{R}, \Gamma>, \quad \text { and } \quad C_{\Gamma}=C_{\Gamma}^{L}-C_{\Gamma}^{R}
$$

If $C_{\Gamma} \neq 0$, then $<\Gamma_{1}^{L} \circ_{1} \Gamma_{2}^{L}, \Gamma>\neq 0$ and $<\Gamma_{1}^{R} \circ_{2} \Gamma_{2}^{R}, \Gamma>\neq 0$. Hence, there exist two insertion data $\pi_{L}$ and $\pi_{R}$ such that $\Gamma_{\pi_{L}} \cong \Gamma \cong \Gamma_{\pi_{R}}$. Moreover, it follows from Lemma 3.4 that

$$
\begin{gathered}
L_{\Gamma_{1}^{L}, \Gamma_{2}^{L}}^{\Gamma}=\left|\mathcal{D}_{\Gamma}\right|=\frac{\left|A u t\left(\Gamma_{1}^{L}\right)\right| \cdot\left|A u t\left(\Gamma_{2}^{L}\right)\right|}{\left|A u t\left(\Gamma, \pi_{L}\right)\right|} \\
R_{\Gamma_{1}^{R}, \Gamma_{2}^{R}}^{\Gamma}=\left|\mathcal{D}_{\Gamma}\right|=\frac{\left|A u t\left(\Gamma_{1}^{R}\right)\right| \cdot\left|A u t\left(\Gamma_{2}^{R}\right)\right|}{\left|A u t\left(\Gamma, \pi_{R}\right)\right|}
\end{gathered}
$$

Thus

$$
\begin{gathered}
C_{\Gamma}^{L}=\frac{L_{\Gamma_{1}^{L}, \Gamma_{2}^{L}}^{\Gamma_{2}}}{\left|\operatorname{Aut}\left(\Gamma_{1}^{L}\right)\right| \cdot\left|A u t\left(\Gamma_{2}^{L}\right)\right|}=\frac{1}{\left|\operatorname{Aut}\left(\Gamma, \pi_{L}\right)\right|} \\
C_{\Gamma}^{R}=\frac{R_{\Gamma_{1}^{R}, \Gamma_{2}^{R}}^{\Gamma}}{\left|\operatorname{Aut}\left(\Gamma_{1}^{R}\right)\right| \cdot\left|A u t\left(\Gamma_{2}^{R}\right)\right|}=\frac{1}{\left|A u t\left(\Gamma, \pi_{R}\right)\right|}
\end{gathered}
$$

Now it remains to show that if $\Gamma_{\pi_{L}} \cong \Gamma_{\pi_{R}}$, then

$$
\left|A u t\left(\Gamma, \pi_{L}\right)\right|=\left|A u t\left(\Gamma, \pi_{R}\right)\right|
$$

In fact both automorphism groups equal $A u t(\Gamma)$. In order to prove this, note that there are (natural) restriction monomorphisms,

$$
\operatorname{Aut}\left(\Gamma_{1}^{L}\right) \times \operatorname{Aut}\left(\Gamma_{2}^{L}\right) \leftarrow \operatorname{Aut}(\Gamma) \rightarrow \operatorname{Aut}\left(\Gamma_{1}^{R}\right) \times \operatorname{Aut}\left(\Gamma_{2}^{R}\right)
$$

since $\Gamma_{i}^{L}$, and respectively $\Gamma_{i}^{R}$, are invaried as being (unique) maximal left normal subgraphs.

Lemma 3.5. We have $\operatorname{Aut}(\Gamma, S)=\operatorname{Aut}(\Gamma, \pi)$, where $S$ is the domain of $\pi$ and $\operatorname{Aut}(\Gamma, S)$ is the subset of automorphisms of $\Gamma$ which invary $S$, i.e. $\Phi(S) \subset S$.

Proof. It is enough to prove " $\subset$ ", since the other inclusion follows from the definition of $\operatorname{Aut}(\Gamma, \pi)$. If $\Phi \in \operatorname{Aut}(\Gamma)$ and $\Phi(S) \subset S$ then

$$
\Phi(s \rightarrow t)=\Phi(s) \rightarrow \Phi(t)=s^{\prime} \rightarrow \Phi(t)
$$

Since $S$ has the property that any of its points has a unique arrow towards $V_{2}$, the vertices of $\Gamma_{2}$, then $\Phi(t)=\pi\left(s^{\prime}\right)=\pi(\Phi(s))$, i.e. $\pi \Phi=\Phi \pi$ on $S$, and therefore $\Phi$ invaries $\pi$.

Now the unique factorization implies that the "Galois group" $A u t(\Gamma, \pi)$ is the full automorphism group.

Lemma 3.6. We have Aut $(\Gamma, \pi)=\operatorname{Aut}(\Gamma)$.

Proof. Let $\pi$ be the left insertion data yielding $\Gamma$ as a left extension (by unique factorization). If $\Phi \in \operatorname{Aut}(\Gamma)$ not only $\Phi$ invaries $\Gamma_{1}$ and $\Gamma_{2}$, but also $S$, the domain of $\pi$ as being the set of arrows lending on the left leg of $\Gamma_{1}$. By the previous lemma, $\Phi$ invaries $\pi$.

Therefore $\operatorname{Aut}\left(\Gamma, \pi_{L}\right)=\operatorname{Aut}(\Gamma)=\operatorname{Aut}\left(\Gamma, \pi_{R}\right)$ is the stabilizer of the action and the normalized coefficients are trivial or equal to 1.

This concludes the proof of Theorem 3.1. 


\section{Conclusions and further developments}

We proved the existence of a canonical solution of Maurer-Cartan equation in the pre-Lie algebra of Lie admissible graphs, "on the nose", without assuming the Jacobi identity holds.

The main fact used in the proof is the unique factorization property enjoyed by graph insertions.

In this way a canonical solution is obtained, which is not surprising, in view of the Hausdorff Lie series, which lies at the core of almost all quantization prescriptions. This opens a pertinent question, the investigation of its relation with the other "universal solution", the Haussdorf series, living on the "base space".

It is also natural to look for a physical interpretation of our solution as a (semi-classical part of the) correlation function in the spirit of [3]. The lack of circuits means in physics jargon finding a solution at "tree level". This does not involve the quantum corrections due to circuits, and relates to the effective action (see also [5]).

Although we investigate the linear case corresponding to Lie admissible graphs, we introduced new tools which, we conjecture, may provide a proof of the general case.

For linear Poisson structures the structure of the Galois group of a left (right) extension is simpler (subobject of the fibered product of $\operatorname{Aut}\left(\Gamma_{1}\right)$ and $A u t\left(\Gamma_{2}\right)$ ), since $\pi$, the insertion data, is injective at the level of interior points, and a permutation of $S$ is equivalent to an inverse permutation of $T$. Nevertheless the "simplification" entailing the left-right symmetry (equal coefficients) seams to be due to the lack of circuits, rather than, as one might expect, from the one-incoming arrow property satisfied by the Lie admissible graphs ( $\pi$ injective on interior points).

We believe that these are interesting topics for further study, revealing some of the relationship between the mathematics and physics of quantum phenomena.

\section{References}

[1] F. Akman, L. M. Ionescu, and P. A. Sissokho. On deformation theory and graph homology. J. Algebra, 2006 (to be published); Preprint math.QA/0507077

[2] A. S. Cattaneo. Formality and star products. Lecture notes by D. Indelicato. Preprint math.QA/0403135.

[3] A. S. Cattaneo and G. Felder. A path integral approach to the Kontsevich quantization formula. Comm. Math. Phys. 212 (2000), 591-611; Preprint math.QA/9902090.

[4] A. Connes and D. Kreimer. Renormalization in quantum field theory and the Riemann-Hilbert problem I: The Hopf algebra structure of graphs and the main theorem. Comm. Math. Phys. 210 (2000), 249-273; Preprint hep-th/9912092.

[5] D. Fiorenza. An introduction to the Batalin-Vilkovisky formalism. Preprint math.QA/0402057.

[6] D. Fiorenza and L. M. Ionescu. Graph complexes in deformation quantization. Lett. Math. Phys. 73 (2005), 193-208; Preprint math.QA/0505410 (extended verison).

[7] S. Gutt. An explicit *-product on the cotangent bundle of a Lie group. Lett. Math. Phys. 7 (1983), 249-258.

[8] S. Gutt. Variation on deformation quantization. Preprint math.DG/0003107, v1.

[9] M. Gerstenhaber and S. D. Schack. Algebraic cohomology and deformation theory. In "Deformation Theory of Algebras and Structures and Applications", M. Hazewinkel and M. Gerstenhaber, Eds. Kluwer Acad. Publ., Dordrecht-Boston-London, 1988, pp. 11-264.

[10] J. Huebschmann and J. Stasheff. Formal solution of the master equation via HPT and deformation theory. Preprint math.AG/9906036.

[11] V. Kathotia. Kontsevich's universal formula for deformation quantization and the Campbell-BakerHausdorff formula, I, Internat. J. Math. 11 (2000), 523-551; Preprint math.QA/9811174.

[12] D. Kreimer. On the Hopf algebra structure of perturbative quantum field theories. Adv. Theor. Math. Phys. 2 (1998), 303-334. 
[13] D. Kreimer. Combinatorics of (perturbative) Quantum Field Theory. Phys. Rept. 363 (2002), 387424; Preprint hep-th/0010059.

[14] M. Kontsevich. Deformation quantization of Poisson manifolds, I. Lett.Math.Phys. 66 (2003), 157216; Preprint hep-th/9709040.

[15] L. M. Ionescu. Perturbative quantum field theory and integrals on configuration spaces. Preprint hep-th/0307062.

[16] L. M. Ionescu. Cohomology of Feynman graphs and perturbative Quantum Field Theory. In "Focus on Quantum Field Theory" , O. Kovras, Ed. Nova Publishers Inc., 2004 ISBN: 1-59454-126-4; Preprint math.QA/0506142

[17] L. M. Ionescu. Perturbative Quantum Field Theory and $L_{\infty}$-Algebras. In "Advances in Topological Quantum Field Theory", J. Bryden Ed. Proceedings of the NATO ARW on New Techniques in Topological Quantum Field Theory, Kluwer Academic Publishers, 2004, pp. 243-252.

[18] L. M. Ionescu. A combinatorial approach to coefficients in deformation quantization. In "Annals of Bucharest University, Proceedings of the G. Vranceanu conference", G. Pripoae, Ed. 2005, pp. 77-96; Preprint math.QA/0404389.

[19] L. M. Ionescu. Perturbative QFT and integrals over configuration spaces. Preprint hep-th/0307062.

[20] L. M. Ionescu. Nonassociative algebras: a framework for differential geometry. Int. J. Math. Math. Sci. 2003, No. 60, 3777-3795; see also Preprint math.DG/9910016.

[21] M. Polyak. Quantization of linear Poisson structures and degrees of maps. Lett. Math. Phys. 66 (2003), 15-35; Preprint math.GT/0210107.

Received December 21, 2006

Revised May 29, 2007 